\title{
El rendimiento académico del alumnado autóctono y de origen inmigrante en la Región de Murcia
}

The academic performance in secondary education of non-immigrant students and those of immigrant origin within the Region of Murcia

\author{
Andrés Escarbajal Frutos \\ e-mail: andreses@um.es \\ Universidad de Murcia. España \\ Juan Navarro Barba (iD \\ e-mail: juan.navarro2@um.es \\ Universidad de Murcia. España \\ Pilar Arnaiz Sánchez \\ e-mail:parnaiz@um.es \\ Universidad de Murcia. España
}

\section{Resumen}

El presente estudio analiza el rendimiento académico en Educación Secundaria del alumnado autóctono y de origen inmigrante escolarizado de $1^{\circ}$ de la ESO a $2^{\circ}$ de Bachiller en la Región de Murcia. Se trata de una investigación cuantitativa no experimental, de corte descriptivolongitudinal, llevada a cabo con 1262 alumnos. Los resultados muestran que el rendimiento académico del alumnado inmigrante es más bajo que el del alumnado autóctono, sin embargo, los alumnos inmigrantes que llegan a $4^{\circ}$ de la ESO y a $2^{\circ}$ de Bachiller obtienen un rendimiento similar o a veces superior al alumnado autóctono. Otro resultado de interés es el referido a las calificaciones obtenidas por el alumnado inmigrante de primera y segunda generación, ya que muestran que el alumnado de primera generación presenta un rendimiento académico superior al de segunda generación. Finalmente indicar que el título de la ESO lo obtiene un porcentaje mayor del alumnado autóctono frente al de origen inmigrante

Palabras clave: rendimiento académico; educación; migrante; extranjero; estudiante.

\section{Abstract}

The present study analyzes the academic performance in Secondary Education of non-immigrant students and those of immigrant origin studying in the $1^{\text {st }}$ and $2^{\text {nd }}$ year of Compulsory Secondary Education and in the $2^{\text {nd }}$ year of a Level Studies within the Region of Murcia. It is non-experimental, quantitative, short longitudinal descriptive study carried out with 1262 students. From the results obtained, the highlight is that the academic performance of immigrant students is lower than that of non-immigrant students, however, immigrant students who complete fourth of Compulsory Secondary Education and complete the $2^{\text {nd }}$ year of Level Studies obtain a similar or sometimes higher level than non-immigrant students. Another result of interest refers to the grades obtained by first and second generation immigrant students, since they show that the first generation students display an academic performance higher than those from the second generation. Finally, it indicates that the Certificate of Secondary Education is obtained by a higher percentage of nonimmigrant students than those of immigrant origin.

Keywords: academic performance; education; migrant; foreign; student.

Cómo referenciar este artículo / How to reference this article:

Escarbajal Frutos, A., Navarro Barba, J., \& Arnaiz Sánchez, P. (2019). El rendimiento académico del alumnado autóctono y de origen inmigrante en la Región de Murcia. Tendencias Pedagógicas, 33, 5-17. doi: $10.15366 /$ tp2019.33.001 


\section{Introducción}

En esta época en la que nos ha tocado vivir se están produciendo cambios radicales a un ritmo vertiginoso, capaces de replantear la relación del hombre (en términos antropológicos) con el mundo, con la cultura, con los demás y consigo mismo. Es una etapa comparable a lo que en su momento significaron el Neolítico o la Revolución Industrial, por poner dos ejemplos. Para la educación estos cambios reclaman un giro copernicano en sus planteamientos que dé respuesta a las diferencias culturales, lingüísticas, políticas, religiosas..., de un mundo interconectado por múltiples vías. En este sentido, la escuela debería ser, sobre todo, un espacio de encuentro, de comunicación y de convivencia en el que sea más importante el diálogo intercultural que las metodologías didácticas novedosas y tecnológicas (Arnaiz, 2012; Cambi, 2010). A nuevo mundo, nuevas categorías para leerlo (Rovatti, 2008). De ahí la urgencia de una escuela intercultural que sea diseñada pedagógicamente para una nueva ciudadanía (Escarbajal, 2015).

Cabría aplicar, pues, el concepto gramsciano de praxis que integra en una misma acción teoría y práctica. O, como dice Zapata-Barrero (2013, p.5), "mostrar que existe una teoría detrás de una práctica intercultural..., pasar de un período de retórica de la interculturalidad a otro más de acción" que ayude al éxito de todos los estudiantes y evite los problemas en el rendimiento académico que muchos de ellos tienen que afrontar, como es el caso del alumnado de origen inmigrante (ÁlvarezSotomayor, Martínez-Cousinou y Gutiérrez-Rubio, 2015). Es evidente que en el momento actual predomina mucho más la palabra que la práctica en la interculturalidad y que, como sostienen Beach y Dovemark (2009), las escuelas y los centros de educación secundaria se están convirtiendo en incubadoras de individualismo competitivo y de movilidad individual, lo que no contribuye para nada a una educación equitativa y de calidad.

Los centros educativos no pueden (ni deben) sustraerse a esta realidad, pues se ven profundamente afectados por lo que Habermas (2000) llamó "la explosión de la globalización y la multiculturalidad" (p. 90). Dado su poder de transformación y su enfoque restaurativo (Roldán y Cantillo, 2012), sus prácticas de mediación entre iguales y sus estrategias didácticas grupales deberían ser favorecedoras de la interculturalidad al mejorar ésta el clima relacional, la convivencia, el respeto y el diálogo. Por eso, las escuelas y los institutos están llamados a ejercer nuevos planteamientos, porque la sociedad sigue manteniendo la firme convicción de que muchos de los problemas sociales actuales podrían ser atenuados o solucionados si se sabe cómo educar desde estas instituciones educativas básicas. Como consecuencia de ello, los docentes y los pedagogos parece que se han visto sorprendidos al tener que reconsiderar su tradicional labor de transmisión de normas, valores, contenidos..., y tener que adaptarse a la nueva situación, a veces, improvisando las respuestas socioeducativas (Portera, 2006).

En el caso de España, a partir de 1990, en época de bonanza económica, los flujos migratorios convirtieron al país en receptor de inmigrantes, hasta el punto de que el $12 \%$ de la población española llegó a ser extranjera (Portes y Aparicio, 2013). De este modo, el número de alumnos extranjeros pasó de sólo un $0,7 \%$ de alumnos matriculados en colegios españoles a multiplicarse por 14, hasta llegar al 10\%. Más de 85.000 nuevos alumnos ingresaron al año en las aulas españolas, casi la mitad procedentes de Centroamérica y Sudamérica, un 30\% de algunos países de Europa del Este, fundamentalmente de Rumanía; y cerca del $20 \%$ de África, sobre todo Marruecos. De Asia el porcentaje fue del 4,8\%. De esos alumnos, prácticamente cuatro de cada cinco (560.000 frente a 120.000) estaban matriculados en la enseñanza pública. La desproporción era evidente, pero las proyecciones no eran mejores para la escuela pública porque la desproporción no dejó de crecer. Por ejemplo, según datos del propio Ministerio de Educación Ciencia y Desarrollo (MECD, 2010), hubo un aumento anual de alumnos inmigrantes de 1,3 puntos en la escuela pública frente a un aumento del 0,5 en la privada. De estos porcentajes y cifras, quizás lo más positivo sea que cuatro de cada cinco alumnos de hijos de inmigrantes no abandonara el sistema escolar, dato que se amplía muy significativamente para la "segunda generación", que se eleva hasta el 90\% (Portes y Aparicio, 2013).

Sin embargo, y a pesar de la importancia de los números, la diversidad cultural no se vio reflejada 
en la legislación legal española hasta finales de la década de los noventa. De hecho, la categoría "alumnado inmigrante" aparece vinculada a la atención a la diversidad impulsada desde la LOGSE en 1990 para la integración de los grupos desfavorecidos y para compensar sus carencias debido al 'déficit' de estos alumnos por distintos motivos (Márquez y García-Cano, 2012). Es con la LOE cuando ya no se relaciona directamente la condición de extranjero con necesidades de compensación (García, García y Moreno, 2012). Se avanzó, pero todavía hace pocos años algunos autores (García-Cano, Márquez y Agrela, 2008) observaron que aumentaba la preocupación en las escuelas ante las nuevas necesidades y demandas (aprendizaje del idioma, superar el déficit curricular y adquirir categorías culturales españolas) del alumno culturalmente diferente al autóctono no siempre bien resueltas.

Así encontramos que el rendimiento educativo del alumnado español está por debajo de la media europea (Calero y Oriol, 2013; OCDE, 2006; Rahona y Morales, 2012), siendo el alumnado de origen inmigrante el que obtiene peores resultados. En lo que se refiere al éxito educativo de los hijos de inmigrantes, hay estudios contradictorios. Si diferenciamos entre alumnado autóctono e inmigrante, encontramos que los alumnos autóctonos obtienen mejores calificaciones que los inmigrantes aunque las diferencias no son significativas siempre (Pereira, Santos Rego y Lorenzo, 2013). Algunos de estos trabajos han demostrado que los resultados son más altos en este colectivo de alumnos que en el de los autóctonos (Angulo, 2003; Ayuntament de Barcelona, 2005; Izquierdo, 2002; García-Nieto, 2004) y que su permanencia en el sistema es también mayor. "Los extranjeros residentes en España tienen de media 8,75 años de estudio, es decir, un 1,15 más que el conjunto de la población residente en el país" (Bancaja, 2005, p. 3).

Si nos centramos en la Región de Murcia, el propio Ministerio de Educación, Cultura y Deportes (2016) nos informa del bajo rendimiento académico de su alumnado, puesto que se sitúa entre las cinco comunidades autónomas españolas con peores resultados en varias de las disciplinas evaluadas. En concreto, en lectura ocupa la cuarta posición empezando por la cola y la tercera en matemáticas en ese mismo orden. Dados estos resultados en el presente estudio queremos indagar acerca de dichos resultados. Para ello estudiaremos el rendimiento académico del alumnado autóctono y de origen inmigrante, aún en los de segunda generación, tanto en la etapa de la Educación Secundaria Obligatoria (ESO) como en Bachiller, en un periodo crítico en nuestra comunidad como es del curso 2007-2008 al curso 2012-2013. A su inicio llegaron muchos alumnos extranjeros pero al final del mismo empezaron a marcharse las familias a sus países de origen debido a la crisis económica de nuestro país y a la mejor situación de los países de procedencia (Ecuador, Bolivia y Colombia).

Analizaremos, pues, las peculiaridades de este periodo gracias a que es la primera vez que se tienen datos del mismo en la Consejería de Educación y que nos han sido facilitados. El presente estudio nos permitirá conocer el rendimiento académico en este periodo así como la promoción e idoneidad del alumnado al final de la ESO, ya que aún hoy en día somos herederos de lo acontecido en ese momento.

Los objetivos que nos planteamos con este fin son los siguientes:

- Objetivo general

Conocer el rendimiento académico y la promoción del alumnado autóctono y de origen inmigrante de la Región de Murcia en Educación Secundaria.

- Objetivos específicos

1. Analizar el rendimiento académico obtenido en lengua y matemáticas por el alumnado autóctono y de origen inmigrante de $1^{\circ}$ de la ESO, $4^{\circ}$ de la ESO y $2^{\circ}$ de Bachiller.

2. Comparar el rendimiento académico obtenido en lengua y matemáticas por el alumnado de origen inmigrante de $1^{\circ}$ de la ESO, $4^{\circ}$ de la ESO y $2^{\circ}$ de Bachiller según primera y segunda 
generación.

3. Conocer los alumnos autóctonos y de origen inmigrante que titulan así como su idoneidad.

\section{Metodología}

\subsection{Diseño}

Se trata de un estudio en el que se recogieron datos en tres momentos de la escolarización del alumnado: a) $1^{\circ}$ de la ESO; b) $4^{\circ}$ de la ESO; y c) $2^{\circ}$ de Bachiller. Así, el diseño de la investigación puede ser catalogado como cuantitativo no experimental, concretamente un diseño de corte descriptivo y longitudinal. A este respecto conviene recordar que los estudios descriptivos tienen la finalidad de exponer o explicar alguna actividad relevante del presente o pasado propia del ámbito socioeducativo (Hernández y Maquilón, 2010).

\subsection{Participantes}

El número de participantes en el presente estudio fue de 1.262 alumnos escolarizados en la Etapa de la Educación Secundaria Obligatoria (ESO) y en Bachiller, en diferentes Institutos de la Región de Murcia pertenecientes a los municipios con mayor población inmigrante (Murcia, Cartagena, Torre Pacheco, Lorca y San Pedro del Pinatar), unos españoles autóctonos y otros de origen inmigrante.

Los españoles autóctonos fueron 663 alumnos (369 chicos y 294 chicas), pertenecientes a 5 centros de mayor escolarización de alumnado de origen inmigrante, y los alumnos de origen inmigrante fueron 599 alumnos (330 varones y 269 mujeres), la totalidad de los alumnos que en 2017 había escolarizados en la Región de Murcia en $1^{\circ}$ de la ESO, siendo un 49\% procedentes de Ecuador, Colombia, Argentina, Bolivia, Chile, Perú, República Dominicana, Paraguay y Uruguay; un 42\% procedentes de Marruecos, Argelia, Mauritania, Malí y Senegal; un 5\% procedentes de Alemania, Reino Unido, Francia, Italia, Portugal, Islandia, Bulgaria y Polonia; un 3\% del resto de Europa y Europa del este y un 1\% de China, Rusia, Armenia, Georgia y Pakistán). Destacar que un 91\% son provenientes de África e Hispanoamérica, por lo que podemos decir que el alumnado de origen inmigrante de la Región de Murcia proviene de una "inmigración económica"; lo que equivale a decir que su entorno socio-familiar es desfavorecido.

\subsection{Instrumento}

Para la recogida de información se hizo uso de la base de datos de la aplicación informática PLUMIER de la Consejería de Educación de la Comunidad Autónoma de la Región de Murcia, la cual permite la obtención de datos de los alumnos con las características comentadas anteriormente. El registro donde se organizó la información de dicha base de datos puede verse en el anexo A

\subsection{Procedimiento}

Los momentos o fases de la investigación, tomando como referencia la clasificación propuesta por Nieto (2011), fueron:

\section{FASE 1: Identificar y delimitar el problema de investigación}

- Pregunta de investigación y planteamiento del problema.

- Revisión bibliográfica y estado de la cuestión en torno a la educación intercultural

- Definición de los objetivos y las variables de la investigación.

FASE 2: Diseño de la investigación e instrumento

- Definición del diseño de la investigación y muestra

- Y diseño del registro de recogida de información.

FASE 3: Recogida de información y análisis de datos

- Recogida de datos de la muestra seleccionada.

- Informatización de la información extraída y análisis de datos.

FASE 4: Presentación y discusión de resultados 
- Descripción y discusión de los resultados.

- Conclusiones.

- Aportaciones, limitaciones e implicaciones educativas.

FASE 5: Informe de investigación

\subsection{Análisis de datos}

- Redacción del informe de investigación de la tesis doctoral.

La elección del tipo de análisis más apropiado para este estudio estuvo en función de las posibilidades de la base de datos y de los objetivos planteados en la investigación. Por ello, se utilizó la estadística descriptiva (Cuesta y Hernández, 2009).

\section{Resultados}

Para dar respuesta a los objetivos de la investigación, los resultados se presentarán siguiendo el orden de los objetivos específicos formulados.

Objetivo 1. Analizar el rendimiento académico obtenido en lengua y matemáticas por el alumnado autóctono y de origen inmigrante de $1^{\circ}$ de la ESO, $4^{\circ}$ de la ESO y $2^{\circ}$ de Bachiller.

En la figura 1 se aprecian los datos de los alumnos aprobados en lengua y matemáticas del grupo de alumnos autóctonos y del grupo de alumnos de origen inmigrante. Se observa que en el curso $1^{\circ}$ de la ESO, en el grupo de autóctonos aprueban a final de curso la lengua el 77\% frente al 55\% de los alumnos de origen inmigrante (22\% de diferencia); en cuanto a las matemáticas aprueban el $72 \%$ de los autóctonos y el 47\% de los de origen inmigrante (un 25\% menos). Luego el éxito escolar en lengua y matemáticas del alumnado de origen inmigrante es un 23,5\% menor que el de los alumnos autóctonos.

Figura 1

Calificaciones en lengua y matemáticas del alumnado autóctono y de origen inmigrante

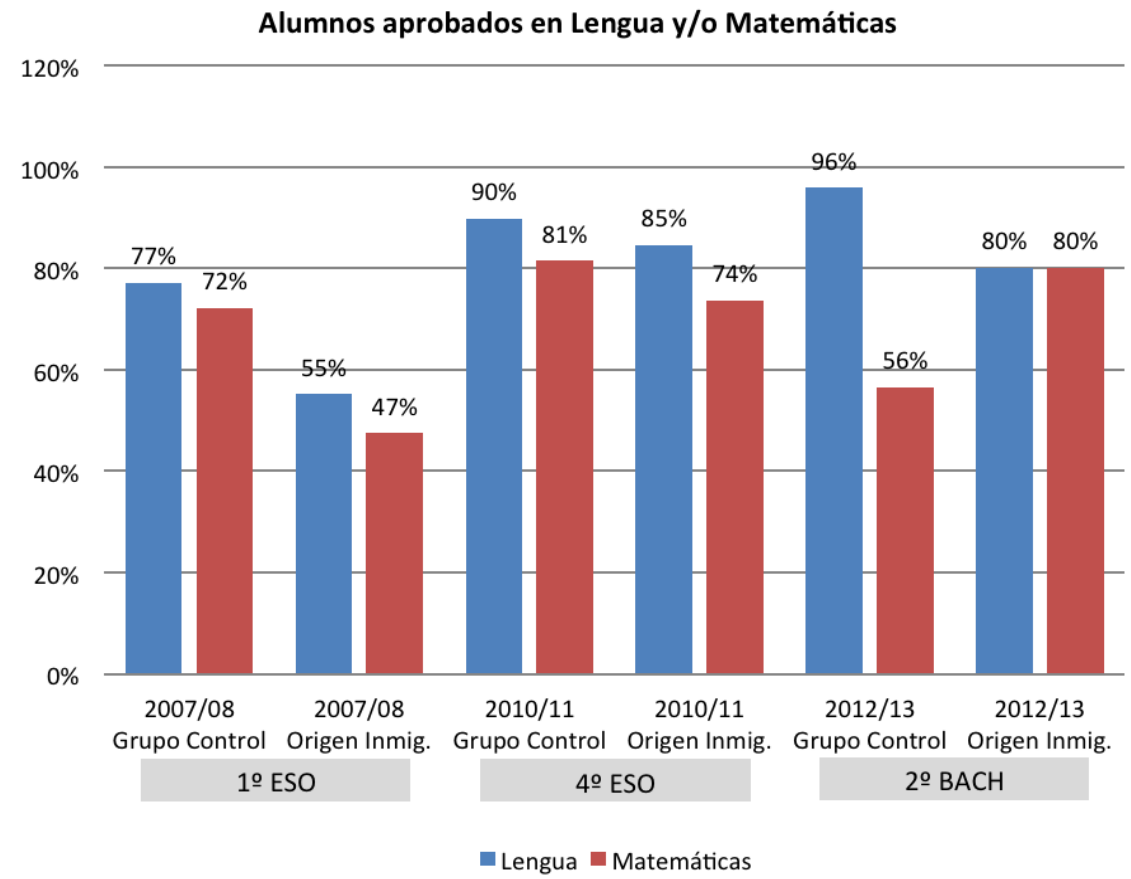

Fuente: Elaboración propia.

Cuatro cursos después terminan $4^{\circ}$ de la ESO el 54\% de los alumnos autóctonos y el 18\% de los de origen inmigrante. El 90\% de los autóctonos aprueba lengua, frente a un 85\% de los de origen inmigrante (5\% de diferencia); y el $81 \%$ de los alumnos autóctonos aprueba matemáticas, frente al 
$74 \%$ de los de origen inmigrante (7\% de diferencia). Por consiguiente, la diferencia en el éxito escolar en lengua y matemáticas del alumnado de origen inmigrante es un $6 \%$ menor que el de los alumnos autóctonos, apreciándose un cambio importante respecto a lo que ocurría en $1^{\circ}$ de la ESO.

Dos cursos más tarde, al final de $2^{\circ}$ de Bachiller, solo llegan el 39,5\% de los alumnos autóctonos y el $9 \%$ de los de origen inmigrante. De éstos, el $96 \%$ de los autóctonos aprueba la lengua frente al $80 \%$ de los de origen inmigrante (16\% de diferencia). Sin embargo, un 56\% de los alumnos autóctonos aprueban las matemáticas frente al $80 \%$ de los alumnos de origen inmigrante, lo que supone un $24 \%$ a favor de éstos últimos. Si hacemos la media de las materias resulta un $4 \%$ a favor de los alumnos inmigrantes, debido a las diferencias obtenidas en la materia de matemáticas, lo que por primera vez pone a los alumnos inmigrantes delante de los alumnos autóctonos.

Objetivo 2. Comparar el rendimiento académico obtenido en lengua y matemáticas por el alumnado de origen inmigrante de $1^{\circ}$ de la ESO, $4^{\circ}$ de la ESO y $2^{\circ}$ de Bachiller según primera y segunda generación.

En cuanto a los alumnos que participaron encontramos que en $1^{\circ}$ de la ESO el 88,1\% del alumnado de origen inmigrante era de "primera generación", y un 11,9\% lo era de "segunda generación". Cuatro años después, de los que continuaron su trayectoria académica, aumentan al $93,4 \%$ los de "primera generación", mientras que disminuyeron un 50\% los de "segunda generación", pasando a ser un 6,6\%. Esa misma tendencia se mantiene en $2^{\circ}$ de Bachiller, pasando a un $96,9 \%$ los de "primera generación" y disminuyendo otro $50 \%$ los de "segunda generación", que pasan a ser un 3,1\%. Por tanto, en principio, haber nacido en España ("segunda generación") no supone mejor adaptación al sistema educativo.

Si comparamos el rendimiento académico por generaciones, encontramos que en $1^{\circ}$ de la ESO los alumnos de "primera generación" (los que nacieron en el país de origen de los padres) aprueban lengua en un porcentaje del 39\% (figura 2). Éstos son alumnos de integración tardía que se incorporaron, más de la mitad de ellos, al sistema educativo con desconocimiento de español. Los alumnos de origen inmigrante de "segunda generación" (nacidos en España siendo hijos de padres inmigrantes) tienen mejores resultados en lengua, con un 54\% de aprobados (un 15\% superior). Igualmente ocurre en matemáticas, los de "primera generación" tienen un 36\% de aprobados frente a un $48 \%$ de los de "segunda generación" (un 12\% superior). Por tanto, de forma global, los datos arrojan un $13,5 \%$ a favor de la "segunda generación".

Figura 2

Comparativa aprobados en lengua y matemáticas entre alumnos de origen inmigrante de "primera" y "segunda generación"

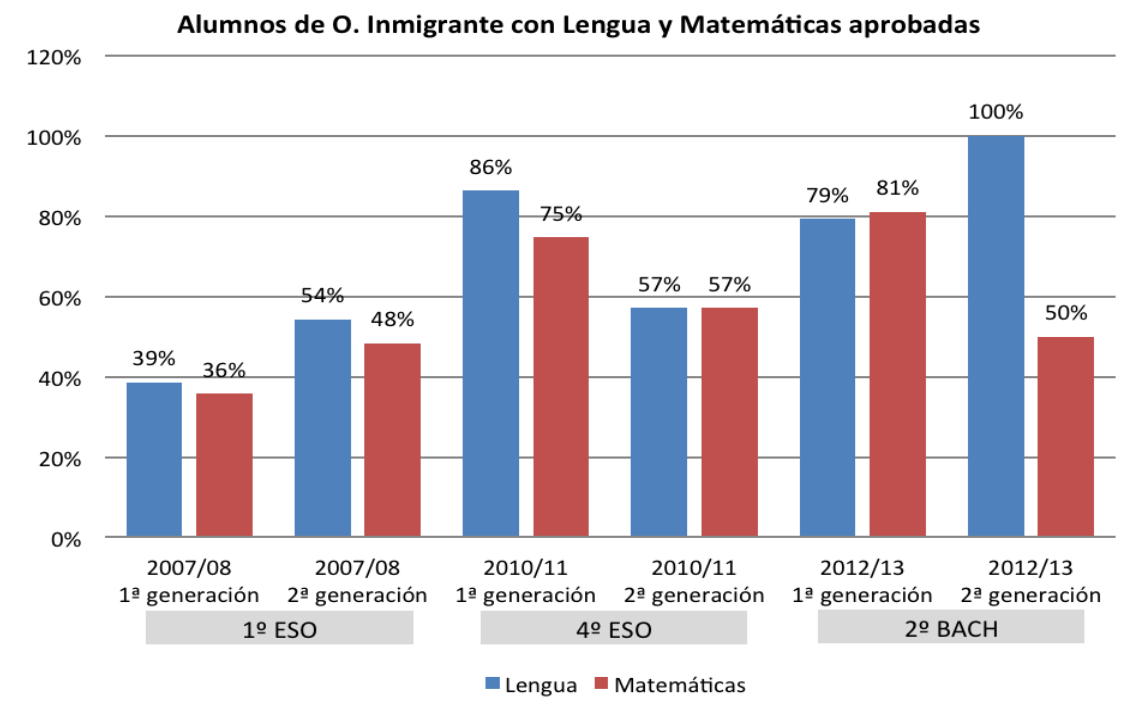

Fuente: Elaboración propia. 
Cuatro años después, al llegar a $4^{\circ}$ de la ESO, los alumnos de "primera generación" tienen un 86\% de aprobados en lengua, y los de "segunda generación" un 57\%; curiosamente se invierten los términos, los de "primera generación" superan en un $29 \%$ a los de la "segunda". Algo similar ocurre en matemáticas, los alumnos de "primera generación" obtienen un $75 \%$ de aprobados frente a un 57\% de los de "segunda generación" (un 18\% inferior). Así, de manera general la media es de un $23,5 \%$ a favor de la "primera generación"

A final de $2^{\circ}$ de Bachiller, los alumnos de "primera generación" obtienen un 79\% de aprobados en lengua, y los de "segunda generación" un 100\% (un 21\% superior); sin embargo, en matemáticas los alumnos de la "primera generación" obtienen un 81\% de aprobados, frente a un 50\% de los de la "segunda generación" (un 31\% inferior). La media de estas puntuaciones es de un 5\% a favor de la "primera generación".

\section{Objetivo 3. Conocer los alumnos autóctonos y de origen inmigrante que titulan así como su idoneidad.}

Se entiende por alumnado que titula aquel que termina el curso o etapa de la ESO y que aprueba todas las materias o le queda alguna sin aprobar, pero la junta de evaluación lo promociona.

Si analizamos el total de los datos, de los alumnos que empezaron $1^{\circ}$ de la ESO (663 autóctonos y 599 de origen inmigrante), el 49\% de los alumnos autóctonos titulan en el primer año de llegada a $4^{\circ}$ de la ESO, frente al 16\% del alumnado de origen inmigrante (figura 3 ).

Figura 3

Alumnos autóctonos y alumnos de origen inmigrante que titulan

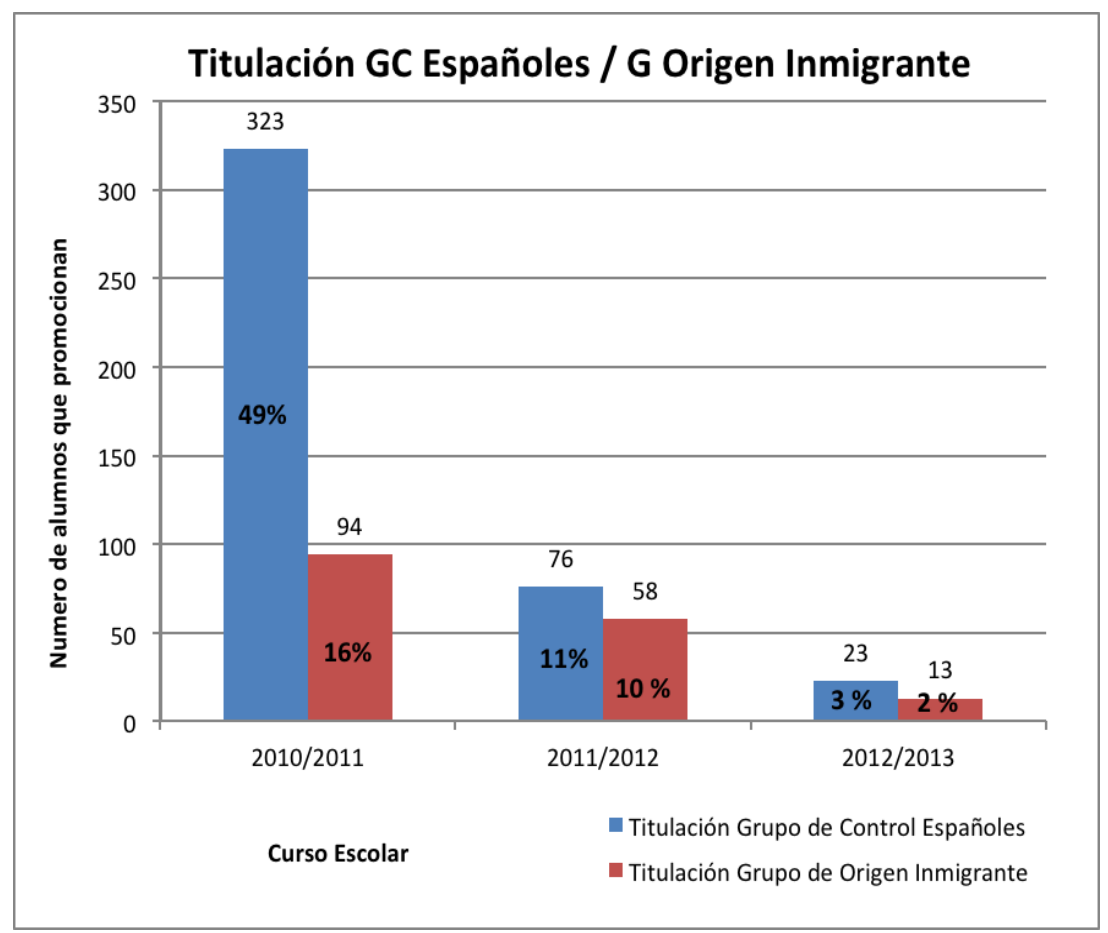

Fuente: Elaboración propia.

Después del primer año de repetición de $4^{\circ}$ de la ESO se observa que consigue titular un 11\% más de los alumnos autóctonos y un 10\% de los alumnos de origen inmigrante. Y un año después, tras un año más de repetición, titulan un 3\% más de alumnado autóctono frente a un $2 \%$ de alumnos de origen inmigrante. Por consiguiente, tras tres años de intento de titulación, lo consiguen un $63 \%$ de alumnos autóctonos y un $28 \%$ de alumnos de origen inmigrante. Estos datos ponen de manifiesto que titula un $44 \%$ menos de alumnado de origen inmigrante respecto del autóctono. 
El análisis del alumnado de origen inmigrante que titula por generaciones (figura 4) evidencia que, en los mismos tres años analizados anteriormente, al finalizar por primera vez $4^{\circ}$ de la ESO, titula el 15\% del alumnado de origen inmigrante que han nacido en el país de origen de los padres, ("primera generación") frente a un escaso 1\% que representan los alumnos de origen inmigrante nacidos en España, (“segunda generación”).

Figura 4

Alumnos de origen e inmigrante, por generación, que titulan

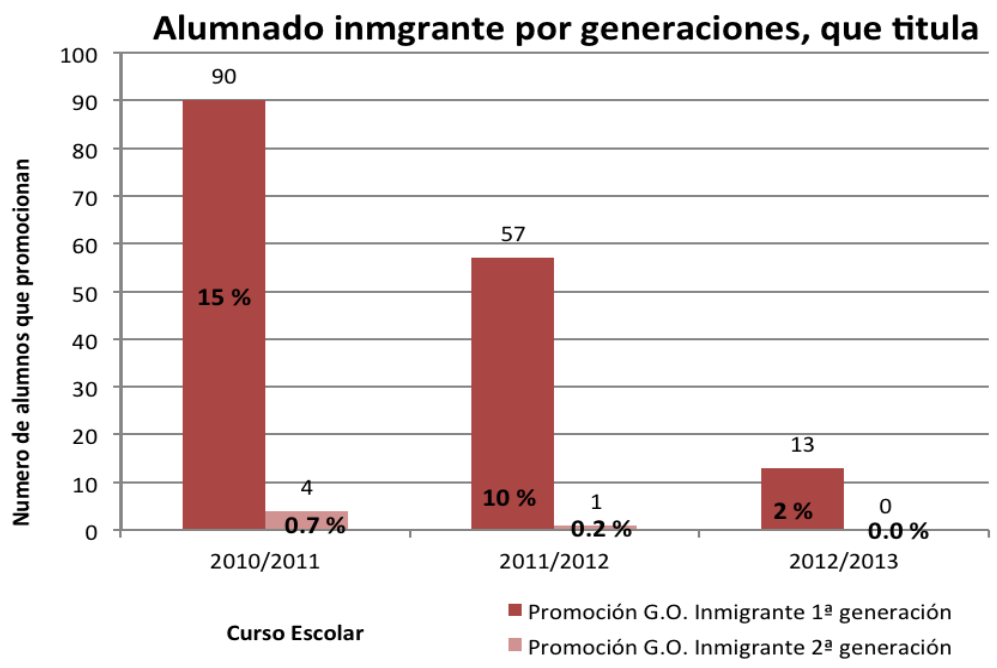

Fuente: Elaboración propia.

En los dos cursos siguientes, tiempo máximo en el que estos alumnos pueden repetir, aparece un $10 \%$ de éxito (titulan) en el primer año de alumnos de origen inmigrante nacidos fuera de nuestras fronteras, frente a un escasísimo $0.2 \%$ de nacidos en España (“segunda generación”). Un año más tarde, en Bachiller, titulan un $2 \%$ de los de "primera generación" y no hay alumnos que titulen de "segunda generación". De todos estos datos cabe significar que, del 28,2\% del alumnado de origen inmigrante que titula, el 27\% corresponde a los alumnos nacidos en el país de origen de los padres (primera generación”) y solamente el 1,2\% a los nacidos en España (“segunda generación”).

En lo que respecta a la idoneidad del alumnado participante, se puede observar en la siguiente figura la correlación entre la edad cronológica y el curso de escolarización.

Figura 5

Idoneidad del alumnado de origen inmigrante

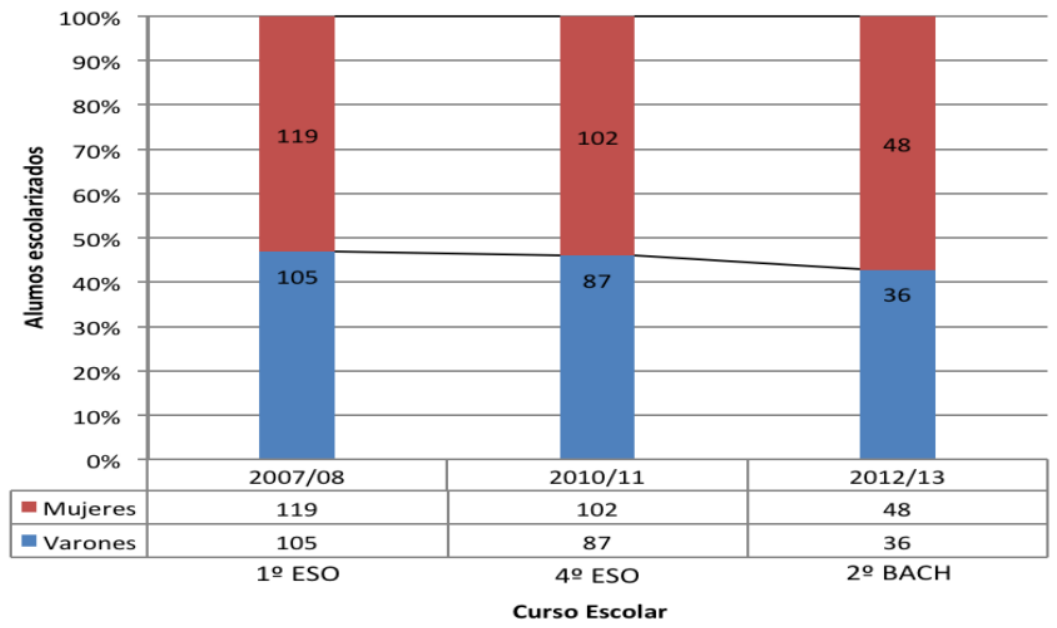

Fuente: Elaboración propia. 
Como se puede comprobar en la figura 5, en el curso 2007/2008, cuando inician la ESO, el 37,4\% del alumnado tiene una adecuación entre su año de nacimiento y curso escolar (idoneidad), mientras que casi dos tercios del alumnado habrá repetido un curso, al menos, al acabar la escolaridad obligatoria. En cuanto a diferencia de género, el 44\% de las alumnas cumplen la idoneidad frente a un $31 \%$ de los alumnos. Este porcentaje es muy similar $(36 \%)$ al que promocionará sin repetir a $2^{\circ}$ de la ESO.

En el curso 2010/2011, cuando corresponde terminar la ESO, de los alumnos que quedan escolarizados, el 47,4\% cumple la idoneidad, tienen 16 años al llegar a $4^{\circ}$ de la ESO. De ellos el 53\% corresponde a las alumnas frente al $42 \%$ de los alumnos.

\section{Discusión y Conclusiones}

Como se ha podido comprobar en los datos expuestos en el primer objetivo, el rendimiento académico, especialmente del alumnado de origen inmigrante, varía a lo largo de los tres momentos estudiados. Así encontramos que en $1^{\circ}$ de la ESO las diferencias entre el alumnado autóctono y de origen inmigrante fueron evidentes en términos generales, observándose una diferencia del $23,5 \%$ a favor del alumnado autóctono. Estos datos coinciden con los obtenidos por García-Borrego (2011) cuando afirma que las notas que consiguen los alumnos de origen inmigrante son inferiores a los de los alumnos autóctonos. La misma tendencia se observa en el Informe PISA (MECD, 2016) y en las investigaciones llevadas a cabo por Kao y Thompson (2003), Pereira, Santos y Lorenzo, 2013 y la OCDE (2006). Lorenzo, Santos y Godás (2012) concluyen en su estudio que las calificaciones son, en general, ligeramente superiores en el alumnado autóctono y que el número de repetidores es superior en el alumnado de origen inmigrante (40,5\%, frente a un 24,8\% del alumno autóctono).

Un hecho destacable en nuestra investigación viene dado por las calificaciones obtenidas por el alumnado en $4^{\circ}$ de la ESO y, especialmente, en $2^{\circ}$ de Bachiller. Si bien es cierto que se observa una disminución tanto del alumnado autóctono como de origen inmigrante que llega a $4^{\circ}$ de la ESO y a $2^{\circ}$ de Bachiller, es importante destacar que cada vez el alumnado de origen inmigrante obtiene mejores calificaciones y sus porcentajes de éxito se acercan más a los del alumnado de origen autóctono. Tanto es así que la diferencia del 23,5\% de $1^{\circ}$ de la ESO queda reducida a un $6 \%$ en $4^{\circ}$ de la ESO. Y lo que más llama la atención: en $2^{\circ}$ de Bachiller se invierte la pirámide y en la asignatura de matemáticas el alumnado de origen inmigrante supera las calificaciones del alumnado autóctono en un 24\%, lo que no había sucedido en los periodos anteriores, determinando que la media de las calificaciones sea de un $4 \%$ a favor del alumnado inmigrante en este nivel.

Estos resultados se enmarcan en recientes investigaciones tales como la llevada a cabo por Cebolla (2013) que justifica esta tendencia a través de la llamada paradoja del optimismo inmigrante: a igualdad de condiciones, los hijos de inmigrantes suelen ser más ambiciosos y esperan más del sistema educativo que los autóctonos, esforzándose en obtener mejores resultados. En esta misma línea, diversas investigaciones han demostrado que los resultados son más altos en el colectivo de alumnos de origen inmigrante que en el de los autóctonos (Angulo, 2003; Ayuntament de Barcelona, 2005; Bancaja, 2005; Izquierdo, 2002; García-Nieto, 2004). Somos conscientes de que estos resultados requieren un estudio de mayor calado, donde puedan ser analizadas otras variables que sabemos que influyen en el rendimiento académico del alumnado tales como las condiciones socioeconómicas de las familias, el nivel de estudios de los padres o la organización de los centros, informaciones no disponibles en la base de datos utilizada, por lo que sugeriremos a la Consejería de Educación de nuestra comunidad la mejora de la misma. El periodo estudiado coincide con los años más duros de la crisis, que siempre tiene unos efectos más crudos en los más desfavorecidos, lo que podría constituir una nueva línea de investigación.

Respecto al segundo objetivo, cabe indicar que las calificaciones obtenidas por el alumnado de origen inmigrante, desagregadas por primera y segunda generación, arrojan unos resultados coincidentes con los que aparecen en el Informe PISA 2015 (MECD, 2016) y en otras investigaciones en $1^{\circ}$ de la ESO, adquiriendo unas características diferentes en $4^{\circ}$ de la ESO y $2^{\circ}$ de 


\section{Bachiller.}

Así vemos que en las puntuaciones existe un porcentaje del 13,5\% a favor del alumnado de origen inmigrante de la "segunda generación" en $1^{\circ}$ de la ESO, lo que coincide con los resultados obtenidos por bastantes trabajos (Álvarez-Sotomayor, Martínez-Cousinou y Gutiérrez-Rubio, 2015; Pereira, Santos-Rego y Lorenzo, 2013; Rodríguez Izquierdo, 2010). Los mismos sostienen que el alumnado inmigrante nacido en España conoce el idioma, está más cercano a la cultura del país de acogida, las expectativas de los padres sobre sus estudios son altas ya que ven en ello una posibilidad de mejorar socio-económicamente y de que se cumplan los sueños que han depositado en el país de acogida, factores todos ellos que se consideran que influyen en la obtención de mejores puntuaciones para esta segunda generación.

Pero en $4^{\circ}$ de la ESO, por el contrario, las calificaciones que obtiene el alumnado de "primera generación" superan en un 23,5\% de forma global a los de la "segunda" y en un 5\% en el caso de $2^{\circ}$ de Bachiller. Estos datos no coinciden con las calificaciones que alumnos obtuvieron en $1^{\circ}$ de la $\mathrm{ESO}$, y contrastan con lo afirmado por las investigaciones anteriores y por lo indicado en el Informe PISA de 2009 (MECD, 2010). Un ejemplo de ello es cuando se expone en el mismo que, en Educación Secundaria, en competencia matemática los alumnos de origen inmigrante de "primera generación" alcanzan 436 puntos y los de "segunda generación" 457 (21 puntos más).

Los resultados obtenidos en nuestro estudio se asemejan, en cambio, a los de Heath, Rothon y Kilpi (2008), cuando plantean que los alumnos de "segunda generación" obtienen resultados académicos inferiores a los de "primera generación", poniendo como ejemplo a los estudiantes marroquíes de Bélgica y Holanda y a los magrebíes de Francia. De igual forma, estos autores indican que, tanto los ingleses de origen indio como los de origen chino, en el Reino Unido superan en rendimiento académico a la mayoría nativa. Kao y Thonson (2008) y la OCDE (2006) documentan en sus trabajos esta misma circunstancia.

Calero, Choi y Waisgrais (2010) analizaron variables como repetición de curso, situación laboral de los padres, nivel de estudios de las familias y lengua hablada en el hogar, diferenciando entre los alumnos de "primera generación" y los de "segunda". En su trabajo no apreciaron diferencias significativas en los resultados académicos de la primera y segunda generación, no fracasaba una más que la otra, lo que justifican aclarando que es posible que el efecto del fracaso escolar sea absorbido por variables correlacionadas con el mismo, tales como la situación económica, la categoría profesional o el capital humano. También pusieron de manifiesto en este trabajo que la presencia de muchos alumnos de origen inmigrante en un mismo centro incrementa el riesgo de fracaso escolar, sobre todo, cuando la concentración supera al $20 \%$ del alumnado total, circunstancia que se daba en diversos centros estudiados.

Encontramos, por tanto, en nuestro estudio que los alumnos de origen inmigrante de "segunda generación", que se entiende que deberían obtener mejores calificaciones que los de "primera generación" y obtener el graduado (titular) al final de $4^{\circ}$ de la ESO, no lo consiguen. Sus resultados y su promoción son menores, lo que llama la atención enormemente. Los datos nos hacen pensar que el sistema educativo criba al alumnado según criterio de rendimiento académico, debiéndose continuar estudiando por ello el papel de los centros en este proceso así como otra serie de variables que puedan estar influyendo en ello (Castejón, Gilar, Miñano y Veas, 2016; Gutiérrez, Tomás, Barrica y Romero, 2017; Ritaco y Amores, 2016).

Uniendo esto con el tercer objetivo de nuestra investigación hemos encontrado que, tras los tres años que es posible permanecer en $4^{\circ}$ de la ESO, finalmente titula el $63 \%$ de los alumnos autóctonos frente al $28 \%$ de los alumnos de origen inmigrante (44\% menos), lo que supone una gran pérdida de estos últimos. Según las estadísticas del MECD (2013), el 21,5\% del alumnado autóctono abandonaba en Educación Secundaria, mientras que se duplicaba el abandono entre los alumnos de origen inmigrante ya que alcanzaban un porcentaje del $43 \%$, cifra bastante parecida a la encontrada en este estudio. Si analizamos esos datos de manera pormenorizada encontramos que, al finalizar $4^{\circ}$ de la ESO, la primera vez llegaron el $54 \%$ de los alumnos autóctonos y de ellos titularon 
el 49\%, y de los alumnos de origen inmigrante llegó casi la mitad pero solo tituló el 16\%.

Estos datos deberían promover una reflexión en las comunidades educativas de los centros, en la sociedad en general y, especialmente, en las Administraciones Educativas, a fin de cumplir con los principios del derecho a la educación para todos los niños proclamados en la Constitución y demás leyes de educación. Más que una acusación de unos a otros, debería ser un esfuerzo y compromiso responsable de todos, siendo conscientes de que los logros o sufrimientos también tendrán que ser compartidos en un futuro próximo.

A modo de conclusión, señalamos que los resultados obtenidos al comparar el rendimiento académico en lengua y matemáticas del alumnado autóctono y con el de origen inmigrante revelan variaciones de interés que requieren seguir siendo estudiadas. La variabilidad de resultados obtenidos al final de $4^{\circ}$ de la ESO y $2^{\circ}$ de Bachiller, entre el alumnado autóctono y de origen inmigrante, así como entre los de primera y segunda generación de este grupo, denota una realidad peculiar y de interés que debe seguir siendo investigada.

También es preciso continuar estudiando en profundidad el rendimiento académico del alumnado inmigrante en su conjunto y en los diferentes niveles de la Educación Secundaria, tanto obligatoria como postobligatoria como por generaciones. Las características encontradas en nuestra Comunidad Autónoma invitan a ello para que se pueda comprender mejor lo que está sucediendo en la misma. Como se ha expuesto, existen unas peculiaridades que no hemos encontrado en otras comunidades autónomas de nuestro país y consideramos de interés seguir indagando sobre ello.

A su vez, somos conscientes de las limitaciones de este trabajo ya que la base de datos utilizada no nos ha permitido estudiar otras variables que pueden estar asociadas al rendimiento académico y, por tanto, a los resultados obtenidos como son el nivel socioeconómico de los alumnos, los estudios de sus padres, características del centro, diferencias entre chicos y chicas, etc. Aparte de estas consideraciones, también sería de interés realizar una réplica de este estudio eligiendo otras cohortes de estudiantes de $1^{\circ}$ de la ESO a $2^{\circ}$ de Bachiller y comprobar si se aprecian resultados similares a los obtenidos en esta investigación o cambian las tendencias, lo cual daría una información clave respecto a los resultados encontrados que permitiría avanzar en el fenómeno estudiado.

\section{Referencias}

Álvarez-Sotomayor, A.; Martínez-Cousinou, G. \& Gutiérrez-Rubio, D. (2015). Cuando la segunda generación no se queda atrás: evidencias sobre el rendimiento académicos de los hijos de inmigrantes en el caso andaluz. Estudios sobre Educación, 28, 51-78.

Angulo, C. (2003). La población extranjera en España. Madrid: INE.

Arnaiz, P. (2012). Escuelas eficaces e inclusivas: cómo favorecer su desarrollo. Educatio Siglo XXI, $30(1), 25-44$.

Ayuntament de Barcelona (2005). La població estrangera a Barcelona, gener 2005. Barcelona: Dpto d'Estadística de l'Ayuntament de Barcelona.

Bancaja (2005). Migraciones, capital bumano y caracteristicas de los municipios. Madrid: Capital Humano.

Beach, D. \& Dovemark, M. (2009). Making right choices: An ethnographic investigation on creativity and performativity in four Swedish schools. Oxford Review of Education, 35, pp. 689-704.

Calero, J. \& Oriol, J. (2013). El rendimiento del alumnado de origen inmigrante en PISA 2012. En INEE, PIS A 2012. Programa para la evaluación Internacional de los alumnos. Informe español. Volumen II: Análisis secundario (pp. 4-31). Madrid: INEE.

Calero, J., Choi, A. \& Waisgrais, S. (2009). Determinantes del rendimiento educativo del alumnado de origen nacional e inmigrante en PISA 2006. Cuadernos económicos de ICE, 78, pp. 281-310. Recuperado de http://www.revistasice.com.pdf

Cambi, F. (2010). Cittadinanza e globalizzazione. Una sfida educativa e pedagogica. En A. Portera, P. Dusi \& B. Guidetti (Ed.), L'educazione interculturale alla cittadinanza. La scuola come laboratorio (pp. 29-34). Roma: Carocci.

Castejón, J. L.; Gilar, R.; Miñano, P. \& Veas, A. (2016). Identificación y establecimiento de las características motivacionales y actitudinales de los estudiantes con rendimiento académico 
menor de lo esperado según su capacidad (underachievement). European Journal of Education and Psychology, 9, pp. 63-71.

Cebolla, H. (2013). Sobre la desventaja educativa de la población inmigrante: ¿Es útil la educación en la diversidad? En R. Zapata-Barrero \& G. Pinyol (Eds.), Manual para el diseño de políticas interculturales (pp. 95-102). Barcelona: Universidad Pompeu Fabra.

Cuesta, J. D. \& Hernández, F. (2009). Métodos cuantitativos de investigación. En P. Colás, L. Buendía \& F. Hernández (Coord.), Competencias científicas para la realización de una tesis doctoral: Guía metodológica de elaboración y presentación (pp. 63-96). Barcelona: Davinci Continental.

Escarbajal, A. (2015). Comunidades Interculturales y Democráticas. Madrid: Narcea.

García, R., García, J.A., \& Moreno, I. (2012). Estrategias de atención a la diversidad cultural en educación. Madrid: Catarata

García Borrego, I. (2011). La difícil reproducción de las familias inmigrantes. ¿Hacia la formación de un proletariado étnico español? Papers, 96(1), pp. 55-76.

García-Cano, M., Márquez, E \& Agrela, B. (2008). Cuando, por qué y para qué la educación intercultural. Discursos y praxis de la educación intercultural. Papers, 89, pp. 147-167

García-Nieto, A. (2004). Los inmigrantes en la Región de Murcia 2002. Murcia: Consejería de Trabajo y Política Social.

Gutiérrez, M.; Tomás, J. M.; \& Barrica, J. M. (2017). Influencia del clima motivacional en clase sobre el compromiso escolar de los adolescentes y su logro académico. Enseñanza \& Teaching, 35, pp. 21-37.

Habermas, J. (2000). La constelación postnacional. Ensayos Políticos. Barcelona: Paidós.

Heath, A., Rothon, C., \& Kilpi, E. (2008). The Second Generation in Western Europe: Education, Unemployment and Occupational Attainment. Annual Review of Sociology, 34, pp. 211-235.

Hernández, F. \& Maquilón, J. J. (2010). Introducción a los diseños de investigación educativa. En S. Nieto (Coord.), Principios, métodos y técnicas para la investigación educativa (pp. 110-126). Madrid: Dykinson.

Izquierdo, A. (2002). La educación errante. En J. Iglesias (Coord.), La sociedad. Teoría e investigación empirica (pp. 207-230). Madrid: Centro de Investigaciones Sociológicas.

Kao, G. \& Thompson, J. S. (2003). Racial and Ethnic Stratification in Educational Achievement and Attainment. Annual Review of Sociology, 29, pp. 417-442.

Lorenzo, M., Santos Rego, M. A. \& Godás, A. (2012). Inmigración y educación. ¿Influye el nivel educativo de los padres en el rendimiento académico de los hijos? Revista Teoría de la Educación, 24(2), pp. 129-148.

Márquez, E., \& García-Cano, M. (2012). Educación intercultural y comunidades de aprendizaje. Madrid: Los Libros de la Catarata.

MECD, Ministerio de Educación, Ciencia y Deporte (2010). Informe estadístico 2010. Madrid. Servicio de Publicaciones del Ministerio de Educación, Cultura y Deportes.

MECD, Ministerio de Educación, Ciencia y Deporte (2013). Informe estadístico 2013. Madrid: Servicio de Publicaciones del Ministerio de Educación, Cultura y Deportes.

MECD, Ministerio de Educación, Ciencia y Deporte (2016). PIS A 2015. Informe español. Ministerio de Educación Ciencia y Desarrollo (MECD). Boletín EducaINEE, Instituto Nacional de Evaluación Educativa, $\mathrm{n}^{\circ}$ 51. Madrid. Recuperado de: http://www.mecd.gob.es/dctm/inee/boletines/educainee51 provokk.pdf?documentId=0901e7 $2 \mathrm{~b} 8228 \mathrm{c} 0 \mathrm{~b} 9$

OCDE (2006). Where immigrants students succeed: A comparative review of performance and engagement in PIS A 2003. Paris.

Pereira, C., Santos Rego, M. A., \& Lorenzo, Mª M. (2013). Alumnos autóctonos y de origen inmigrante en PISA: variables de rendimiento. Revista Galego-Portuguesa de Psicoloxía e Educación, 21(1), pp. 95-109.

Portera, A. (Ed.) (2006). Educazione interculturale nel contesto internazionale. Milano: Angelo Guerini.

Portes, A. \& Aparicio, R. (2013). Investigación longitudinal sobre la segunda generación en España. Madrid. Fundación Ortega-Marañón.

Rahona, M. \& Morales, S. (2013). Diferencias en el rendimiento educativo de nativos e inmigrantes en España. Revista de Asociación Sociológica de la Educación, 6, pp. 72-90.

Ritacco, M. \& Amores, F. J. (2016). Estudiantes en riesgo de exclusión educativa en Secundaria. 
Percepciones del profesorado implicado en programas extraordinarios de prevención del fracaso escolar. Enseñanza \& Teaching, 34, pp. 137-160.

Roldán, C. \& Cantillo, L. (2012). Formas alternativas a la resolución tradicional de conflictos: Las prácticas restaurativas. En B. López \& M. Tuts (Eds.), Orientaciones para la práctica de la educación intercultural (pp. 137-148). Madrid: Wolters Kluwer.

Rovatti, A. (2008). Possiamo addomesticare l'altro? Udine: Forum.

Rodríguez Izquierdo, R. M. (2010). Éxito y fracaso escolar de la primera y segunda generación de estudiantes de origen inmigrante. Estudios sobre Educación, 19, pp. 97-118.

Zapata-Barrero, R. (2013). Del diálogo intercultural a la acción intercultural. En R. Zapata-Barrero \& G. Pinyol (Eds.), Manual para el diseño de politicas interculturales (pp. 5-12). Barcelona: Universidad Pompeu Fabra.

\section{Anexo A. Registro del rendimiento académico según base PLUMIER}

\begin{tabular}{|c|c|c|c|c|c|}
\hline & & & \multicolumn{3}{|c|}{ Seguimiento anual 1} \\
\hline & & & Varones & Mujeres & Total \\
\hline \multicolumn{6}{|c|}{ Número total de alumnos } \\
\hline \multirow{13}{*}{ Escolarización } & \multirow{4}{*}{ ESO } & $1^{\circ}$ & & & \\
\hline & & $2^{\circ}$ & & & \\
\hline & & $3^{\circ}$ & & & \\
\hline & & $4^{\circ}$ & & & \\
\hline & \multirow{2}{*}{ Diversificación } & $3^{\circ}$ & & & \\
\hline & & $4^{\circ}$ & & & \\
\hline & \multicolumn{2}{|r|}{ PCPI } & & & \\
\hline & \multirow{2}{*}{ Bachiller } & $1^{\circ}$ & & & \\
\hline & & $2^{\circ}$ & & & \\
\hline & \multirow{2}{*}{ FP } & $1^{\circ} \mathrm{GM}$ & & & \\
\hline & & $2^{\circ} \mathrm{GM}$ & & & \\
\hline & \multicolumn{2}{|c|}{ ESPA y otras } & & & \\
\hline & \multicolumn{2}{|c|}{ Abandonos (Sin evaluación) } & & & \\
\hline \multicolumn{3}{|c|}{ Idoneidad (nacidos en 1995) } & & & \\
\hline \multirow{2}{*}{ Generación } & \multicolumn{2}{|c|}{ Primera (NIE) } & & & \\
\hline & \multicolumn{2}{|c|}{ Segunda (DNI) } & & & \\
\hline \multicolumn{3}{|c|}{ Alumnos en centros públicos } & & & \\
\hline \multicolumn{3}{|c|}{ Alumnos en centros privados o concertados } & & & \\
\hline \multicolumn{3}{|c|}{ Número de centros públicos } & & & \\
\hline \multicolumn{3}{|c|}{ Número de centros privados o concertados } & & & \\
\hline \multirow{4}{*}{$\begin{array}{l}\text { Asignaturas de } \\
\text { Lengua/Matemáticas }\end{array}$} & \multirow{4}{*}{$\begin{array}{c}\text { Autóctonos/Origen } \\
\text { Inmigrante }\end{array}$} & Suspensos $(0,1,2,3$ o 4$)$ & & & \\
\hline & & Aprobados $(5 \circ 6)$ & & & \\
\hline & & Notables $(7$ o 8$)$ & & & \\
\hline & & Sobresalientes $(9$ o 10$)$ & & & \\
\hline
\end{tabular}

